\title{
Politique
}

\section{Les causes et les remèdes de la « crise " des finances publiques au Québec}

\section{Richard Carter}

Numéro 3, hiver 1983

La crise des finances publiques au Québec

URI : https://id.erudit.org/iderudit/040419ar

DOI : https://doi.org/10.7202/040419ar

Aller au sommaire du numéro

Éditeur(s)

Société québécoise de science politique

ISSN

0711-608X (imprimé)

1918-6584 (numérique)

Découvrir la revue

Citer cet article

Carter, R. (1983). Les causes et les remèdes de la " crise » des finances publiques au Québec. Politique, (3), 91-116. https://doi.org/10.7202/040419ar d'utilisation que vous pouvez consulter en ligne.

https://apropos.erudit.org/fr/usagers/politique-dutilisation/ 


\title{
Les causes et les remèdes de la «crise» des finances publiques au Québec
}

\author{
par \\ Richard Carter
}

École nationale d'administration publique

Cet essai cherche à décrire les principales raisons, économiques et politiques qui expliquent les contraintes budgétaires observées à l'échelle municipale et provinciale au Québec ${ }^{1}$. L'analyse économique de la politique (Public Choice) est utilisée essentiellement pour expliquer les causes politiques des problèmes financiers des gouvernements et pour tracer une esquisse des changements qui pourraient permettre d'éviter le renouvellement d'une telle situation dans l'avenir.

\section{Les causes des restrictions budgétaires au Québec}

Les coupures budgétaires provinciales

Avant de s'interroger sur les causes des restrictions budgétaires au Québec il est important de noter que ce n'est pas

1. Certaines portions de cet essai sont tirées de conférences présentées par l'auteur et d'un article paru dans la revue Commerce. 
l'ensemble des dépenses gouvernementales qui est réduit mais bien le taux de croissance de certains budgets sectoriels.

En effet, de 1976 à 1980 le taux de croissance annuel des dépenses du gouvernement du Québec était en moyenne de 13. $12 \%$. De 1980 à 1981 , ce taux fut de $16.6 \%$ et, selon les résultats préliminaires, il s'est maintenu à $16 \%$ de 1981 à 1982. Si on en juge par les chiffres, le gouvernement devrait arrêter de parler de décroissance puisque c'est justement lorsqu'il se fixe un tel objectif que ses dépenses croissent le plus rapidement!

Mais pourtant, on observe bien des coupures budgétaires qui affectent des secteurs tels que l'éducation et la santé. Pourquoi de telles restrictions? Où va l'argent qui, semble-t-il, est épargné suite aux restrictions budgétaires? La réponse à la deuxième question est très simple. L'argent que l'on obtient par les restrictions budgétaires est dépensé par l'État pour d'autres fins. En particulier, il sert à financer a) les augmentations de salaire réel consenties dans le secteur public lors des dernières conventions collectives, b) le service de la dette qui s'est gonflé considérablement par suite de la hausse des taux d'intérêt c) les dépenses additionnelles d'aide sociale et le manque à gagner fiscal causés par la récession. De telle sorte qu'on observe une croissance accrue de la part des dépenses gouvernementales dans notre économie (les dépenses du gouvernement du Québec augmentèrent de $16.6 \%$ de 1980 à 1981 alors que le produit intérieur brut ne s'est accru que de $10.5 \%$ ) et une réallocation de ces dépenses principalement en faveur des employés du secteur public et du service de la dette.

Certes on ne peut pas tous être d'accord avec le fait que les employés du secteur public gagnent plus cher que leur contrepartie dans le secteur privé (il s'agirait de 10 à $20 \%$ de plus selon diverses sources) et que leurs salaires augmentent plus vite 
que le taux d'inflation et les salaires dans le secteur privé. Mais, même si on accepte l'idée que les fonctionnaires méritent d'être avantagés d'une quelconque façon, on peut se demander pourquoi il faut financer les hausses de salaires par des coupures budgétaires et quelques augmentations des taxes? Ne pourraiton pas augmenter les impôts de façon importante ou emprunter davantage? Après avoir compris qu'il n'y a pas réellement décroissance de l'ensemble des dépenses du gouvernement mais tout simplement déplacement dans les types de dépenses, on doit se demander pourquoi le gouvernement n'a tout simplement pas fait comme auparavant, alors qu'il finançait ses augmentations de dépenses par des emprunts, des taxes ou des transferts provenant du gouvernement fédéral. La réponse peut s'élaborer en trois points.

\section{a) Les transferts}

Premièrement, le gouvernement fédéral depuis quelques temps déjà réduit ses transferts aux provinces. De telle sorte que le gouvernement du Québec ne peut plus financer ses augmentations de dépenses à l'aide des transferts du fédéral. Cette source de financement devient donc moins importante et on ne peut y trouver les fonds nécessaires pour financer les hausses de salaires consenties dans les conventions collectives ainsi que les frais additionnels encourus pour le service de la dette.

\section{b) Les taxes}

Les gouvernements hésitent toujours à augmenter les taxes car cela est particulièrement nuisible pour les élections. De plus, il est crucial de noter que le Québec est la province où les taux moyens de taxation sont les plus élevés au Canada (environ 20\% de plus que la moyenne canadienne). De telle sorte que toute augmentation importante des taxes au Québec peut conduire à l'émigration d'individus et d'entreprises sans compter qu'elle in- 
cite les nouvelles entreprises à s'établir ailleurs qu'au Québec. Au cours de la dernière décennie, près de 200,000 personnes et nombre d'entreprises ont quitté le Québec et, dans son discours budgétaire de mars 1981, le ministre Parizeau a souligné l'importance de ne pas accroître les taxes des entreprises et des individus par crainte de les faire fuir davantage et de réduire les profits déclarés au Québec. La logique est simple, plus vous taxez, moins il reste de personnes et d'entreprises pour payer les taxes de telle sorte que les recettes fiscales présentes et futures ne s'accroissent pas beaucoup et peuvent même diminuer. En d'autres termes, même si le gouvernement pouvait se permettre des hausses importantes de taxes sans compromettre ses objectifs politiques (i.e. réélection) cela modifierait peu ses recettes fiscales et ne règlerait pas ses problèmes financiers.

\section{c) Les emprunts}

Déjà le gouvernement du Québec a fortement utilisé les emprunts. Avec une dette accumulée dépassant $\$ 9$ milliards en 1980 et un déficit de plus de $\$ 3$ milliards pour 1981 , le gouvernement du Québec doit limiter ses emprunts s'il ne veut pas voir diminuer davantage sa cote sur les marchés financiers. Une baisse de cote signifie tout simplement que le gouvernement devra payer des taux d'intérêt encore plus élevés, ce qui représenterait un fardeau additionel pour les générations à venir qui devront payer des taxes supplémentaires afin de rembourser la dette accumulée. Par ailleurs, plusieurs interprètent cela comme étant significatif d'une mauvaise administration financière de la part d'un gouvernement.

C'est parce que le coût du service de la dette est déjà très élevé et parce qu'il a déjà enregistré une diminution de la cote sur ses obligations que le gouvernement semble fermement décidé à ne pas laisser le déficit dépasser $\$ 3$ milliards. Sans compter que les emprunts du gouvernement sur le marché local 
peuvent réduire le financement disponible aux entreprises et retarder la fin de la récession.

On comprend donc maintenant que face aux augmentations de dépenses causées principalement par les conventions collectives passées et par le service de la dette, le gouvernement du Québec a eu recours principalement aux coupures budgétaires et, récemment, aux augmentations des taxes indirectes pour se financer. À court terme, ces mesures furent préférées aux emprunts additionnels comme mode de financement. Dans la mesure où les coupures budgétaires n'ont pas modifié la qualité et la quantité des services de façon importante, cela représentait vraisemblablement la méthode la moins coûteuse du point de vue politique. Cependant, on peut croire que la remise en cause de programmes entiers, si elle s'accompagne de pressions politiques importantes, incitera le gouvernement à effectuer des emprunts additionnels. Malgré les conséquences économiques néfastes de tels emprunts, il faut reconnaître qu'une baisse dans la cote des obligations gouvernementales n'a pas le même poids politique qu'une contestation bien orchestrée sur la colline parlementaire.

Une bonne part des contraintes actuelles aurait pu être évitée si le gouvernement n'avait pas tant emprunté par le passé et s'il avait été moins généreux dans les conventions collectives. Par exemple, un réalignement complet de la rémunération du secteur public sur celle du secteur privé permettrait une économie d'au moins \$1 milliard. Lorsqu'on sait que les compressions budgétaires prévues initialement pour 1981-1982 étaient justement de l'ordre de $\$ 1$ milliard, on est tenté de conclure que les contraintes budgétaires servaient essentiellement à financer un enrichissement démesuré pour les employés du secteur public. De telles statistiques nous indiquent que les contraintes budgétaires au Québec ne sont pas uniquement le résultat de la crise économique nord-américaine. Elles sont le fruit d'une trop 
grande générosité à l'égard des employés du secteur public; générosité qui, selon plusieurs, s'apparente à des intérêts politiques bien circonscrits.

$\mathrm{Si}$ on adopte une perspective politique, la crise québécoise actuelle peut s'interpréter comme le résultat du comportement rationnel de politiciens qui, pour s'attirer des votes, procurent des gains substantiels à des groupes de citoyens bien organisés, en l'occurrence ici les employés du secteur public, et en font supporter le coût à l'ensemble de la population par le biais de taxes indirectes et de restrictions budgétaires uniformes. En somme, les membres d'un groupe bien organisé obtiennent des bénéfices importants dont le coût, étant réparti sur l'ensemble de la population, est minime pour chaque citoyen considéré séparément. ${ }^{2}$

Ce phénomène est apparent aujourd'hui à cause des problèmes financiers qu'il occasionne pour le gouvernement. Il ne faut pas croire, cependant, qu'il ne favorise que les employés du secteur public. En fait, il s'agit là d'une partie intégrante du jeu politique qui occasionne des pertes d'efficacité considérables dans les démocraties modernes. En effet, il suffit d'observer le nombre important de législations dont l'objet est de promouvoir les intérêts économiques de divers groupes en les protégeant de la concurrence locale (corporations professionnelles, syndicats, etc.) et internationale (tarifs, politique d'achat local, etc.) pour comprendre que les avantages concédés aux employés du secteur public représentent un exemple particulièrement visible d'un phénomène plus général.

De la même façon, on peut expliquer l'importance de la dette par des considérations politiques. Les emprunts des gouvernements représentent des taxes différées dont le fardeau repose en partie sur les générations futures. Il est donc plus facile pour

2. Nous reviendrons sur ce sujet dans la troisième section de cet essai. 
un gouvernement de financer un projet qui bénéficie aux votants actuels si les coûts du projet sont supportés par les générations futures qui, elles, ne votent pas. À court terme, une telle approche permet de gagner de nombreux votes et d'en perdre très peu.

Il faut donc reconnaître qu'une part importante des problèmes financiers actuels du Québec résulte du fonctionnement de notre système démocratique. Et qu'au delà des solutions de court terme destinées à rétablir un certain équilibre dans les finances publiques, il faut envisager des solutions politiques de long terme qui réduiraient la facilité avec laquelle un gouvernement peut favoriser certains groupes au détriment de l'ensemble de la population.

\section{Profil financier des municipalités et contraintes budgétaires}

Un survol des finances municipales permet d'élucider comment la conjoncture actuelle aux niveaux provincial et national peut exercer des pressions favorables à l'adoption de contraintes budgétaires à l'échelle municipale.

Premièrement, il faut noter que de 1971 à 1980 le budget des dépenses pour l'ensemble des municipalités est passé de $\$ 961.7$ millions à $\$ 3,220$ millions, pour un taux d'accroissement annuel moyen de $14.4 \%$. Pour la même période, les dépenses per capita ont augmenté à un taux annuel moyen de $14 \%$. Ce taux a finalement atteint $27 \%$ entre 1979 et $1980 .^{3}$ Quant aux revenus, ils sont passés de $\$ 950.4$ millions en 1971 à $\$ 3,384.7$ millions en 1980 , pour un taux de croissance annuel moyen de $15.2 \%$.

3. Le taux de croissance de 1979 à 1980 est gonflé dans les statistiques officielles par une sous-estimation des dépenses de 1979 causée par l'usage d'un exercice financier de huit mois par les villes de Montréal et Québec. Source: Bureau de la statistique du Québec, Finances municipales. 
Dans un tel contexte de croissance, il est difficile de parler d'austérité générale et les contraintes budgétaires dont on parle au niveau municipal peuvent refléter des situations particulières plutôt qu'une situation d'ensemble. De plus, si on suppose que l'ensemble des municipalités imposent aujourd'hui des restrictions budgétaires, le scénario doit alors être similaire à celui de la province, les restrictions sont effectuées dans certains secteurs afin de financer les augmentations de dépenses dans d'autres secteurs d'activité. Dans une telle optique, les principaux facteurs exerçant une pression sur les finances municipales sont les suivants:

a) Les taux d'intérêt

De 1971 à 1980, le service de la dette représentait en moyenne $25 \%$ des dépenses municipales. En 1980, le service de la dette était, après les salaires, la principale dépense pour l'ensemble des municipalités. On comprend donc l'effet dévastateur de la hausse des taux d'intérêt sur les coûts de financement. De 1979 à 1980, les dépenses consacrées au service de la dette augmentèrent de $23.8 \%$ pour l'ensemble des municipalités. En plus de l'effet important de la hausse des taux d'intérêt sur les dépenses municipales, il faut noter qu'une telle augmentation incite l'administrateur à éviter l'emprunt comme source de financement. Si bien qu'il cherche à financer les augmentations de dépenses soit par des taxes additionnelles, soit par des transferts accrus, soit par des restrictions budgétaires sectorielles.

\section{b) Les salaires}

Pour 1980, la somme des salaires, honoraires professionnels et contributions de l'employeur s'élevait à \$1,228.7 millions, soit $38.2 \%$ du budget consolidé de l'ensemble des municipalités. En 1979, cette somme était de l'ordre de $\$ 967$ millions pour une augmentation d'environ $27 \%$ de 1979 à 1980 . Il 
est donc plausible que l'augmentation dans la rémunération des employés constitue, avec le service de la dette, la principale source de l'accroissement des dépenses. Face à de telles augmentations des dépenses, les municipalités peuvent avoir recours aux taxes foncières et aux transferts plutôt qu'aux restrictions budgétaires comme sources de financement. Malheureusement, l'opportunité de financer les dépenses additionnelles par ces méthodes est relativement limitée car: a) la pression fiscale exercée sur les contribuables québécois est déjà très élevée et comme l'impôt foncier représente une taxe très visible, une augmentation subite du taux d'imposition est souvent perçue comme un suicide politique; b) la condition peu reluisante des finances provinciales et fédérales ne laisse pas entrevoir des augmentations salutaires des transferts destinés aux municipalités. Si bien que les municipalités sont incitées à utiliser les contraintes budgétaires pour financer les augmentations de dépenses enregistrées au niveau du service de la dette et de la masse salariale.

c) Structure des revenus et réforme fiscale

Pour les municipalités, le problème du financement des augmentations de dépenses est aggravé par un changement subtil dans la structure de financement. Il s'agit essentiellement de la réduction des transferts par rapport aux revenus de sources locales et le remplacement graduel des transferts inconditionnels par des transferts conditionnels. Ainsi, en 1971, les transferts représentaient $24.4 \%$ des revenus alors qu'en 1980 ils ne constituaient que $9.4 \%$ des revenus consolidés de l'ensemble des municipalités. Par ailleurs, alors que les transferts inconditionnels représentaient $85 \%$ du total des transferts en 1971 , ils ne représentaient plus que $32 \%$ en 1980. La source de ce changement est l'abolition par la réforme fiscale, du transfert de la taxe de vente. Le principal résultat de cette modification sera 
de réduire considérablement la part des transferts inconditionnels et des transferts en général comme source de financement pour les municipalités.

Il y a une compensation certes, par le transfert quasi intégral de l'impôt foncier scolaire aux municipalités, l'élargissement de l'assiette fiscale et le réaménagement des taxes d'affaires, mais il demeure qu'un tel changement, par l'autonomie qu'il consacre, oblige l'administration municipale à plus de vigilance financière. À court terme, il est possible que la réforme fiscale permette à l'administration municipale de substituer $\$ 1$ dollar de transfert perdu par $\$ 1$ dollar d'impôt foncier additionnel. Mais à long terme, les augmentations de revenus ne pourront plus s'obtenir indépendamment des décisions locales concernant l'évaluation foncière et le taux d'imposition. C'est à dire que les revenus ne pourront s'accroittre automatiquement avec les ventes enregistrées dans la province de Québec; il faudra décider, au niveau local, d'accroître les impôts. Cela représente une contrainte politique importante au financement municipal. On peut comprendre que la réforme fiscale permet une plus grand autonomie financière pour les municipalités. Mais une autonomie accrue n'est pas synonyme d'un financement plus aisé. Au contraire, l'importance accrue de l'impôt foncier comme source de financement rendra encore plus visible le coût des services municipaux et il sera difficile, du point de vue politique, d'accroître les dépenses municipales.

En somme, on peut conclure qu'au niveau municipal les problèmes financiers sont relativement similaires à ceux du gouvernement provincial. Les augmentations dans les dépenses occasionnées par les salaires et le service de la dette nécessitent un financement additionnel. L'usage des emprunts est peu recommandé étant donné l'importance actuelle de la dette et le niveau élevé des taux d'intérêt. Par ailleurs, il ne faut pas espérer de miracle du côté des transferts et l'usage de l'impôt 
foncier est toujours restreint par les contraintes politiques. Si bien que malgré leurs inconvénients, les contraintes budgétaires peuvent apparaître comme la meilleure source de financement pour l'administrateur municipal qui doit affronter les augmentations dans la masse salariale et le service de la dette.

Que faire?

Face à la situation qui prévaut au Québec, tant à l'échelle provinciale que municipale, on peut envisager plusieurs types de solutions. Il y a des solutions de court terme touchant la productivité et les politiques salariales des gouvernements et des solutions de long terme qui remettent en question certains secteurs d'activité des gouvernements et les règles du jeu de notre système démocratique.

\section{Les solutions de court terme}

Si, avec de nombreux analystes, on s'accorde pour conclure que des augmentations supplémentaires de taxes accentueraient considérablement les difficultés conjoncturelles de l'économie du Québec, et que le maintien d'une cote favorable pour les titres provinciaux et municipaux est un objectif primordial des gouvernements, il devient clair que les solutions de court terme les plus adéquates sont l'amélioration de la productivité dans différents secteurs d'activité des gouvernements et une politique salariale destinée à rétablir aussi rapidement que possible la parité entre le secteur privé et le secteur public (municipal et provincial).

a) Les gains de productivité et les coupures budgétaires

Les déboires actuels des finances publiques au Québec constituent l'occasion rêvée pour remettre en question l'ensemble des activités gouvernementales. Au lieu de s'apitoyer sur la conjoncture actuelle il faut y voir l'occasion d'améliorer la 
productivité du secteur public et d'en faire un système plus efficace et donc moins onéreux. La politique de contraintes budgétaires pratiquée par les gouvernements peut aider à cet égard, mais elle possède aussi des inconvénients.

Par exemple, imaginons les réactions possibles d'un administrateur public auquel on annonce une coupure budgétaire de quelques millions. Deux possibilités s'offrent à lui. D'une part il peut réagir politiquement. C'est-à-dire qu'il réduira la qualité et la quantité des services essentiels dans l'espoir que la clameur publique rendra les politiciens plus cléments à son égard et qu'ils annuleront les restrictions budgétaires qui lui sont imposées. Ou encore, il accumulera les déficits dans l'espoir que le gouvernement en assumera éventuellement la responsabilité. D'autre part, il peut réagir «économiquement». Dans ce cas, il cherchera à réduire les activités les moins «rentables» ou essentielles dans son organisation. C'est la réaction «économique» qui doit être encouragée. Le problème, c'est que le comportement politique et le comportement économique peuvent être indissociables. Par exemple, il est clair que les organisations publiques qui depuis longtemps étaient gérées de façon efficace sont les plus affectées par les contraintes actuelles. N'étant plus capables de couper dans les services nonessentiels, les administrateurs doivent alors réduire de façon draconienne la qualité et la quantité de leurs services. De telle sorte qu'il est pratiquement impossible dans ces cas de savoir si la réaction de l'administrateur est purement politique (i.e. s'il tente tout simplement de conserver son budget en laissant croire que tous ses services sont efficaces) ou si, réellement, il n'y a plus d'économies à réaliser. Il est navrant de constater que les coupures budgétaires affectent autant les vaches grasses que les vaches maigres et que les institutions qui opéraient de façon efficace par le passé sont pénalisées alors que celles qui jouissaient de nombreux services superflus et de dépenses inutiles 
affrontent aisément les restrictions budgétaires. Dans un tel contexte, l'administrateur est encouragé à effectuer des dépenses inutiles afin de réduire l'impact d'éventuelles contraintes futures; c'est loin d'être une incitation à l'efficacité.

Considérons maintenant la rationalité économique qui devrait guider les choix budgétaires en période de décroissance. Le critère de l'économiste est simple, il s'agit premièrement d'éliminer les services dont les bénéfices n'excèdent pas les coûts et, si cela est insuffisant pour satisfaire à la nouvelle contrainte budgétaire, il faudra alors couper dans les services dont les bénéfices nets sont les moins élevés. Cela signifie premièrement qu'un système de prix de revient soit établi afin de déterminer le coût de différents services internes et externes. Si on veut rationaliser les choix budgétaires, il faut connaître les coûts et bénéfices des services rendus.

Une fois les coûts estimés on peut les comparer avec ceux qui sont enregistrés dans d'autres institutions privées et publiques et voir ainsi comment ils peuvent être réduits. Par exemple, si le coût annuel d'entretien est $\$ 35$. le pied carré pour une institution alors qu'ailleurs ce coût est de $\$ 15$. parce qu'on confie cette tâche à des entrepreneurs privés, on doit alors recommander le rétablissement des sous-contrats pour minimiser les coûts. De la même façon, si le personnel administratif requis pour procurer une unité de service est plus élevé dans une instititution publique (hôpital, école, municipalité) comparativement à une autre procurant des services similaires, il faudrait envisager une réduction dans ce type de personnel.

En plus des comparaisons qui pourraient éclairer plusieurs administrateurs à propos de l'efficacité dans la production de divers services, il existe aussi des mesures incitatives qui peuvent conduire à des économies substantielles. Une telle mesure serait, par exemple, d'établir un système de primes à l'économie. Tout employé qui découvrirait une façon de couper 
les dépenses sans compromettre la qualité et la quantité des services produits pourrait avoir droit à une prime équivalente à un pourcentage de l'économie réalisée. À titre d'illustration, si une économie de $\$ 1,000,000$ par année est réalisée dans l'entretien des rues grâce à un changement technique recommandé par un ingénieur on pourrait récompenser ce dernier par une prime de $\$ 100,000$. Le simple fait de connaitre l'existence d'une telle politique de prime à l'économie peut conduire le personnel à penser en termes d'efficacité et fournir à l'administrateur des idées et des informations qu'il n'aurait jamais découvertes autrement. Alors les ingénieurs du gouvernement deviendraient plus ingénieux au sens de l'économiste.

Finalement, il faut noter que les conventions collectives dans le secteur public ont compartimenté et rigidifié l'allocation des tâches. De telle sorte que les administrateurs n'ont pas la liberté d'action requise pour permettre une réallocation efficace des ressources humaines. Sans compter que les clauses de sécurité d'emploi obligent parfois les gouvernements à payer des salaires confortables à des employés qui sont "sous-utilisés» alors que dans le secteur privé le nombre de chômeurs s'accroît de jour en jour. Pour remédier à cette situation, les gouvernements doivent renégocier les conventions collectives dans l'optique d'une plus grande liberté d'action pour les administrateurs afin que ceux-ci n'aient pas uniquement l'illusion d'administrer mais qu'ils possèdent des pouvoirs réels de réaffectation des ressources physiques et humaines mises à leur disposition.

\section{d) Les politiques salariales}

On a déjà noté qu'à l'échelle provinciale la rémunération des employés du secteur public était plus élevée et augmentait plus rapidement que la rémunération versée pour des emplois équivalents, dans le secteur privé. À moins de supposer que la 
productivité dans le secteur public est plus grande que dans le secteur privé (plusieurs pensent le contraire), un ajustement des rémunérations s'impose. Dans la mesure où une situation similaire est observée dans les municipalités, des procédures d'ajustement devraient être envisagées.

Les solutions à moyen et à long terme

Les mesures citées jusqu'à présent sont destinées à minimiser les coûts d'opération. On se rappellera, cependant, que réduire les coûts d'opération n'est pas l'objectif fondamental de l'administrateur public. Ce qui importe, c'est de produire les services désirés au moindre coût possible. Ici, on aborde le problème le plus important de tout le secteur public. L'économiste préconise qu'on coupe les dépenses là où les bénéfices n'excèdent pas les coûts. Mais comment évalue-t-on les bénéfices? Qui peut évaluer la «qualité» des services et donc les bénéfices pour la société dans des domaines tels que la santé, la justice, l'éducation, les services de police et la protection contre les incendies? Comment effectuer des analyses bénéfices-coûts valables dans des circonstances où la notion «d'intérêt public» fluctue avec les partis politiques en place et les pressions qui s'exercent? L'administrateur public devrait couper ses dépenses dans les secteurs les moins bénéfiques à la société mais la définition des bénéfices est souvent difficile. À cet égard, les municipalités sont avantagées relativement au gouvernement provincial car certains des services qu'elles produisent (aqueduc et égouts, loisirs, enlèvement des ordures, déneigement, voirie, etc.) génèrent des bénéfices qui peuvent être évalués en partie ou en totalité par la tarification des usagers.

Le problème c'est qu'en l'absence de tarification des usagers il est plus difficile de savoir si les bénéfices excèdent les coûts. À chaque fois que j'achète un paquet de gomme à mâcher, par exemple, c'est que le bénéfice que je retire de cet 
achat excède son coût. Comme c'est vrai de tous ceux qui font comme moi, c'est dire que si les revenus de la vente de gomme à mâcher excèdent les coûts de production, il y a un gain net pour la société puisque tous les consommateurs en retirent un bénéfice net. Si le coût d'un produit, qu'il s'agisse de services de loisirs, d'éducation ou d'un paquet de gomme n'est pas payé par les usagers, il est difficile sinon impossible de savoir si les bénéfices retirés par ces derniers excèdent les coûts et donc s'il y a efficacité économique. Du point de vue de l'estimation des bénéfices dérivés de diverses activités, on comprend donc pourquoi il est si important d'utiliser la tarification de l'usager; à bien des égards, c'est la meilleure façon de savoir si les bénéfices excèdent réellement les coûts. Dans la mesure du possible, on doit donc rétablir la tarification des usagers si ce n'est que pour savoir quels sont les services provinciaux et municipaux qui procurent des bénéfices nets importants à la population. On peut alors effectuer des restrictions budgétaires dans les services où les bénéfices nets sont les moindres. On doit aussi comprendre que la tarification des usagers permet désormais l'autofinancement et réduit d'autant les besoins financiers (emprunts et taxes) de la municipalité. Mais, si l'autofinancement est techniquement possible, pourquoi ne pas laisser l'entreprise privée prendre la relève du gouvernement?

Il n'appartient pas seulement aux directeurs d'hôpitaux et aux recteurs d'université de remettre en question certains des services que leurs institutions procurent. Le gouvernement doit lui même questionner ses interventions globales dans l'économie. Par exemple, l'idée du ticket-modérateur a principalement été rejetée à court terme pour des raisons politiques et parce qu'il semble qu'une application mitigée de ce principe ne procurerait pas des gains financiers importants et immédiats pour le gouvernement. À long terme cependant, il est difficile de penser à une amélioration de l'efficacité dans l'allocation des 
ressources gouvernementales sans l'établissement de ticketsmodérateurs dans les secteurs d'activités où cela est possible. Pensons par exemple aux services de santé et à l'éducation. Dans chacun de ces cas, le gonflement des coûts supportés par le gouvernement résulte partiellement de la gratuité universelle qui fut instaurée afin de faciliter l'accessibilité à ces services. Comme ce ne sont pas les usagers qui paient directement pour les services de santé et d'éducation, ils exigent la plus grande qualité et diversité de service pouvant être produites. Si les étudiants payaient une plus grande proportion du coût de leur éducation, ils seraient moins enclins à se spécialiser dans des domaines où déjà il y a surabondance de main-d'œuvre, et nos universités produiraient moins de chômeurs instruits. À la limite, l'objectif d'accessibilité à l'éducation pourrait être maintenu en ne finançant que les usagers à faible revenu et en laissant les institutions d'enseignement se concurrencer pour la clientèle. Dans un tel système de concurrence, les coûts de production diminueraient et les dépenses de l'État seraient limitées à des subventions aux plus démunis.

De la même façon, par l'instauration d'un déductible ou du principe de coassurance dans le secteur de la santé, on pourrait réduire les dépenses gouvernementales et maintenir l'accessibilités aux services par des subventions aux plus démunis. On blâme souvent les médecins pour l'usage abusif de soins et certains pensent solutionner le problème en remplaçant la rémunération à l'acte par le salaire moyen. Cependant, on doit réaliser que c'est parce que ni le médecin ni le patient ne déboursent les coûts réels des services médicaux utilisés que cette surconsommation est observée. Le changement de rémunération conduira uniquement à des files d'attentes plus longues ou à une multiplication des médecins qui ne travaillent qu'une fraction de la semaine tout en retirant un salaire «confortable». Ce qu'il faut, c'est rendre les individus plus conscients des coûts suscités 
par leur consommation de soins de santé et, en présence d'assurances privées et publiques, ceci ne peut se faire que par l'introduction d'une franchise ou d'un principe de coassurance. Comme solution de moindre mal par exemple, on pourrait instaurer un régime où le pourcentage de coassurance est proportionnel au revenu à partir d'un certain seuil de pauvreté. Les plus démunis auraient un usage gratuit des soins de santé alors que ceux dont le revenu est plus élevé débourseraient une fraction positive des coûts. Une autre alternative est celle de l'assurance catastrophe où seuls les frais dépassant un montant donné seraient assurés. Ce montant pourrait être ajusté selon les revenus et permettre une certaine équité. Chose certaine, cette mesure diminuerait le nombre de consultations pour les rhumes et les mini-dépressions. Sans compter qu'elle pourrait susciter une plus grande prévention contre la maladie et les accidents de la part des citoyens.

Sans vouloir reprendre ici le débat sur le bien-fondé de la tarification des usagers, il importe seulement de reconnaitre qu'en l'absence d'une telle tarification il est à toute fin pratique impossible de juger de la désirabilité relative des différents biens et services produits par le secteur public. En situation de gratuité, le système politique est lui-même incapable d'évaluer la valeur des bénéfices retirés par l'ensemble des citoyens puisqu'il n'existe aucune mesure de la valeur attribuée au service par chaque individu. ${ }^{4}$ Tout au plus, le système politique peut enregistrer les pressions de différents groupes et estimer les bénéfices selon l'intensité de ces pressions. À toute fin pratique, cela signifie que les groupes biens organisés et plus susceptibles d'exercer des pressions (associations de producteurs, syndicats, etc.) seront avantagés au détriment des autres groupes (con-

4. Sans prétendre que la valeur sociale d'un bien est la somme des valeurs individuelles, on peut difficilement concevoir la première sans la deuxième. 
sommateurs et payeurs de taxes en général). Les coupures budgétaires affecteront les faibles et épargneront les forts.

Solution à très long terme: le processus politique

On a déjà noté que la dynamique politique n'était pas étrangère aux problèmes financiers rencontrés par les différents niveaux de gouvernement au Québec. Ainsi, l'usage abusif des emprunts et les salaires élevés dans le secteur public peuvent s'expliquer par les gains à court terme qu'obtiennent politiciens et partis politiques en termes de votes. Cela suppose, évidemment, que la recherche des votes est un objectif primordial pour les gouvernements démocratiques, et que ceux-ci ne sont pas essentiellement préoccupés par la poursuite d'un quelconque intérêt public. Cependant, on peut concilier ces deux objectifs de la façon suivante.

Dans un système démocratique il faut qu'un parti politique détienne le pouvoir s'il désire implanter l'idéologie qu'il associe généralement à l'intérêt public. L'élection est alors perçue comme un moyen permettant d'atteindre des fins qui transcendent le moyen. Mais voici, vous ne pouvez jamais dissocier la fin du moyen. L'élection ou la réélection n'est pas uniquement une contrainte à l'atteinte d'un objectif supérieur. À court terme, il s'agit d'un objectif dont l'atteinte est sine qua non pour la réalisation de tout autre objectif. C'est pourquoi, de nombreux puristes idéologiques se dissocient souvent des partis politiques qui reflètent partiellement l'idéologie concernée. L'idéologue se soucie peu des élections alors que pour le parti politique il s'agit là d'un moyen qui prend la dimension d'un objectif et il est prêt à sacrifier certaines positions idéologiques (ou en adopter d'autres) pour s'approprier ou conserver le pouvoir. Si bien que parmi les politiques gouvernementales associées à une idéologie, seules celles qui pourront franchir 
l'équivalent d'un tamis politique seront implantées. Du point de vue politique, une intervention gouvernementale sera donc jugée acceptable si on anticipe qu'elle attirera plus de votes qu'elle n'en fera perdre.

Du moment qu'on reconnait l'importance de cette dynamique où les gouvernements échangent des politiques contre des votes, il convient alors de s'interroger sur le type de politique qui procure le plus de vote à un gouvernement. À cet égard, les analystes du Public Choice présument que ce sont les intérêts privés des citoyens, plus que leur conception personnelle de l'intérêt public qui détermine l'allocation des votes. ${ }^{5}$ Cette motivation personnelle des individus fut décrite éloquemment par Alexis de Tocqueville qui notait: "Ceux-là admettent bien, pour principe général, que la puissance publique ne doit pas intervenir dans les affaires privées; mais, par exception, chacun désire qu'elle l'aide dans l'affaire spéciale qui le préoccupe et cherche à attirer l'action du gouvernement de son côté tout en voulant la resserrer de tous les autres ${ }^{6}$.

La pertinence de cette observation fut maintes fois soulignée par des observateurs de l'histoire politique et économique, tant au Canada qu'aux États-Unis. Du point de vue théorique, elle fut principalement développée et formalisée par Mancur Olson ${ }^{7}$ qui démontra que ce sont généralement les petits groupes de producteurs (industries, syndicats, etc.) dont les coûts d'organisation sont minimes et dont les intérêts économiques peuvent être facilement favorisés par des interventions publiques, qui influenceront les politiques gouvernementales de toutes sortes. L'un des corollaires de cette approche est que les

5. Pour un survol de la littérature, voir C.D. Mueller, Public Choice, Cambridge surveys of economic litterature, London, Cambridge University Press, 1979.

6. Alexis de Tocqueville, De la démocratie en Amérique, Paris, Idées, 1968, 318.

7. Mancur Olson, The Logic of collection Action, Cambridge, Harvard University Press. Cambridge, 1965. 
coûts des dites interventions gouvernementales seront divisés entre un grand nombre d'invidivus de telle sorte que pour chacun d'eux, le fardeau interventionniste reste minime et ne justifie pas un lobbying quelconque pour s'en protéger. ${ }^{8}$

Une deuxième approche théorique pour expliquer les actions gouvernementales consiste à supposer que les objectifs électoraux des politiciens ne représentent pas des contraintes particulièrement sévères et qu'entre les élections, les politiciens et les bureaucrates possèdent des pouvoirs discrétionnaires qui leur permettent de satisfaire leurs propres intérêts personnels. Ce qui est suggéré ici, c'est que l'action gouvernementale n'est pas uniquement dirigée pour satisfaire les demandeurs de «politiques publiques» et qu'elle s'explique en partie par les intérêts des offreurs de ces services. Au niveau de la bureaucratie, cette hypothèse fut principalement élaborée par les travaux de William Niskanen ${ }^{9}$, qui suggéra que les intérêts bureaucratiques convergeaient vers l'objectif de la maximisation des budgets. La formulation la plus générale de ce modèle d'action discrétionnaire de la part des gouvernements demeure cependant celle de J.L. Migué et G. Bélanger ${ }^{10}$ où on suppose que les bureaucrates disposent d'une marge de manœuvre et qu'ils peuvent allouer certaines ressources disponibles de la façon qui maximise leur bien-être sans nécessairement maximiser le budget. Ce modèle permet d'introduire l'influence des idées sur les politi-

8. Une autre version de cet argument consiste à supposer que les coûts de l'activité gouvernementale ne sont même pas perçus par les contribuables. C'est la théorie de l'illusion fiscale qui fut popularisée dans la littérature par J. M. Buchanan, Public Finance in Democratic Process, Chapel Hill: University of North Carolina Press, 1967. Voir aussi Cotton M. Lindsay, "A theory of Governement enterprise", Journal of Political Economy, 84: 1061 (oct. 1976).

9. William Niskanen, Bureaucracy and Representative Government, Chicago, Aldine Atherton 1971.

10. J.L. Migué et G. Bélanger, «Toward a general theory of managerial discretion », Public Choice, 17 (Spring 1974), 27-42. 
ques gouvernementales car la maximisation du bien-être des bureaucrates peut signifier la poursuite d'une idéologie particulière.

En bref, les modèles économiques de l'action gouvernementale peuvent s'assembler en un modèle plus général qui incorpore simultanément l'offre et la demande de politiques. Du côté de l'offre, on peut supposer l'existence d'une bureaucratie monopolistique dont l'offre de services incorpore l'influence des idées et des connaissances scientifiques. Cependant, l'influence des offreurs sur l'activité gouvernementale dépend essentiellement de la demande politique pour ces activités. De telle sorte que l'influence des idéologies et des connaissances scientifiques marquera les activités gouvernementales dans la mesure où elles peuvent être associées aux intérêts spécifiques (souvent économiques et financiers) d'importants groupes de pression. Des observations historiques variées ainsi que des résultats empiriques plus rigoureux semblent confirmer la vraisemblance de ce type de modèle du comportement gouvernemental dans différentes sphères d'activité. 11

Notons finalement que ce modèle du comportement gouvernemental ne nie pas que certains votes soient motivés par des considérations idéologiques ou que différentes décisions gouvernementales soient publiquement justifiées par des raisons d'intérêt collectif; ce que le modèle soutient, c'est que de telles considérations sont insuffisantes pour expliquer les décisions

11. Quelques exemples historiques pour le Canada sont présentés dans $\mathbf{R}$. Carter, "Séparation, Annexion et Fédéralisme: au-delà des préceptes normatifs usuels », à paraître, Actualité économique et "La dérèglementation des communications aux États-Unis: son impact effectif et potentiel sur la règlementation québécoise », Ministère des Communications du Québec, Juin 1982. Des études empiriques ont aussi souligné la pertinence du modèle pour expliquer l'imposition de tarifs au Canada et aux États-Unis. À cet effet, voir J. Pincus, "Pressure groups and the pattern of tariffs", Journal of Political Ecor my, Août 1975 et R.S. Saunders, "The Political Economy of effective tariff protection in Canada's manufacturing Sector", Revue Canadienne d'économique, Mai 1980, 340-48. 
gouvernementales et que, comme dans les marchés privés, ce sont les intérêts économiques de divers groupes qui devanceront la formation d'une idéologie particulière permettant de justifier une décision gouvernementale ou encore sanctionneront une idéologie existante afin d'influencer cette décision.

$\mathrm{Si}$ on reconnaît que la crise actuelle résulte en partie de cette dynamique de notre système politique qui encourage les politiciens à procurer des bénéfices importants à différents groupes de votants et à en faire supporter le coût à l'ensemble de la population, on doit comprendre qu'il existe peu de solutions à court terme. Plusieurs peuvent croire que le problème immédiat serait réglé si on limitait ou interdisait les droits de vote et de grève aux employés du secteur public. Après tout, ne détiennent-ils pas une place privilégiée pour faire pression sur les politiciens et sur la population pour obtenir des bénéfices démesurés? De la même façon que les politiciens sont limités dans l'usage des pouvoirs publics pour faire avancer leurs intérêts privés, ne devrait-on pas limiter le droit de vote des fonctionnaires pour qui l'avancement de l'intérêt «public» se confond facilement avec la croissance du gouvernement?

Le problème avec cette solution c'est qu'elle est trop radicale pour les résultats obtenus. En effet, si on désire limiter l'achat de vote ou le favoritisme électoral pratiqué par nos gouvernements, on peut établir des contraintes plus générales qui ne compromettront pas des droits et libertés considérés comme fondementaux dans notre société. Si, par exemple, la constitution imposait que les rémunérations et modalités d'emploi dans le secteur public évoluent de la même façon que dans le secteur privé, on consacrerait moins d'énergies en négociation des conventions collectives et les coûts imposés à l'ensemble de la population par des grèves dans le secteur public seraient moindres. Des résultats similaires pourraient aussi s'obtenir par une plus grande décentralisation des pouvoirs au niveau local et 
par un usage plus généralisé du référendum dans le processus décisionnel. Si les conventions collectives étaient négociées sur une base sectorielle et locale, il y aurait moins de grèves affectant l'ensemble de la population québécoise. En conséquence, les pressions politiques exercées sur le gouvernement central seraient moindres et le résultat des conventions collectives reflèterait davantage la conjoncture économique plutôt que la conjoncture politique. De la même façon, la décentralisation effective ${ }^{12}$ permet un plus grand usage du référendum comme processus décisionnel et on peut croire que les interventions gouvernementales seraient alors moins dépendantes des intérêts politiques immédiats des législateurs et reflèteraient plus adéquatement les volontés d'une majorité de la population. Autant la concurrence sur les marchés économiques permet de préserver la souveraineté des consommateurs, la concurrence entre les gouvernements locaux peut sauvegarder les intérêts des individus. Il faut diviser les gouvernements pour assurer le règne des citoyens. ${ }^{13}$

On s'est longtemps inspiré de la Suède comme modèle pour justifier nos politiques économiques sociales-démocrates. Mais aujourd'hui, alors que plusieurs analystes considèrent que la Suède frôle la banqueroute ${ }^{14}$ on pourrait peut être faire changement et analyser le modèle politique de la Suisse où, faute de notoriété internationale pour des programmes sociaux d'envergure, le système politique est l'un des plus démocratique et des plus stable au monde et où les «crises» économiques et

12. Le terme décentralisation signifie ici que des pouvoirs fiscaux supplémentaires ainsi que de nouvelles responsabilités décisionnelles en matière de dépenses sont accordés aux gouvernements locaux.

13. Pour un survol de la littérature et une description des avantages et inconvénients économiques du fédéralisme, voir R. Carter "Séparation, Annexion et Fédéralisme", $o p$. cit.

14. Business Week, Juin 1981. 
politiques n'ont pas l'importance ni la fréquence de celles qu'on observe dans notre société.

\section{Conclusion}

L'état déplorable des finances publiques au Québec n'est pas uniquement le résultat de la politique monétaire américaine et de la mauvaise volonté du gouvernement fédéral à augmenter les transferts destinés à la province de Québec. Une cause importante est le comportement rationnel des politiciens qui cherchent à concentrer les bénéfices et à disperser les coûts des activités gouvernementales. Face à ce constat, il faut résister à la tentation d'assigner un tel comportement à un parti politique particulier et réaliser qu'il est la conséquence directe de notre système démocratique. La solution n'est pas de remplacer un parti politique par un autre ou d'imaginer de nouveaux processus de contrôle interne contre le petit patronage; il faut comprendre que le fonctionnement même de notre démocratie est basé sur le patronage à grande échelle, l'achat de votes par des subventions diverses, l'instauration de tarifs à l'importation et des lois multiples protégeant les producteurs contre la concurrence. Lorsque tous les groupes d'intérêts au Québec auront leur petit monopole local, tous les revenus et tous les prix seront plus élevés; aucune richesse n'aura été crée et des ressources économiques auront été gaspillées dans la course aux rentes que permet notre système politique, notre société sera plus pauvre et ses possibilités de développement économique plus limitée.

Il fut un temps ou la simple observation d'une inefficacité des marchés (monopole, externalités, bien publics) était suffisante pour justifier l'intervention des gouvernements. L'expérience de cette intervention doit désormais nous convaincre qu'à l'inefficacité des marchés il faut comparer l'inefficacité des gouvernements avant de recommander toute nouvelle inter- 
vention. Malheureusement, comme toute autre idéologie, la recherche de l'efficacité a peu de poids dans l'arêne politique et on conçoit difficilement comment un système politique peut s'amender lui-même afin de respecter davantage les désirs de la population. Un gain de pouvoir décisionnel pour le citoyen est équivalent à une perte pour le gouvernement central et rares sont les élus et bureaucrates qui volontairement se feront harakiri. 